7. L'Union désire attirer l'attention sur la grande importance pour l'astronomie de la publication des I5 volumes de la nouvelle observation photographique des zones de l'A.G. Du personnel et de l'argent existent en Allemagne pour compléter ce travail, ainsi que pour l'impression et pour la correction des épreuves, mais la publication est retardée par le manque de papier en Allemagne. L'Union espère que les fonds nécessaires à l'achat du papier et à la reliure pourront être trouvés pour la publication.

8. L'Union attire l'attention de ses membres sur l'importance d'obtenir une photocopie des parties nord et sud de la Bonner Durchmusterung et exprime l'espoir que la quantité de papier nécessaire sera mise à la disposition de l'Observatoire de Bonn.

\title{
Commission des Finances
}

La Commission des Finances propose à l'Assemblée Générale:

I. Que les subventions suivantes soient allouées pour la période s'écoulant du I janvier I949 jusqu'au 3 I décembre I95I.*

\section{(a) Subventions annuelles}

Commission

5 (Bibliographie mensuelle) $\quad \ldots \quad \ldots \quad \ldots$

Dollars

6 (Bureau des Télégrammes) $\quad \ldots \quad \ldots \quad \ldots$

ro (Cartes héliographiques de la photosphère) ...

II (Cartes synoptiques) $\quad \ldots \quad \ldots \quad \ldots \quad \ldots$

I9 (Bureau central de la Variation des Latitudes)

20 (Centre des Petites Planètes, à Cincinnati) ...

27 (Liste des Noms d'Etoiles variables) ... ...

27 (Ephémérides des Etoiles doubles à Eclipses)

3 I (Bureau International de l'Heure) ... ...

" $\quad 38$ (Echange des Astronomes) $\quad \ldots \quad \ldots, \quad \ldots$

$\begin{array}{lllllll}\text { Bureau de l'Union } \ldots & \ldots & \ldots & \ldots & \ldots & \ldots & \ldots\end{array}$

\section{(b) Subventions uniques spéciales}

Commission I2 (Tables du Spectre solaire)

" $\quad$ I8 (Publication des Résultats des Longitudes Mondiales I933) $\quad \ldots \quad$... $\quad \ldots \quad \ldots \quad \ldots$

I9 (Publication des Résultats de la Variation des Latitudes jusqu'à la fin de I948) $\quad$... $\quad \ldots$

Ig (Dépenses du Déménagement du Bureau Central $\begin{array}{llllll}\text { à Turin) } & \ldots & \ldots & \ldots & \ldots & \ldots\end{array}$

23 (Carte du Ciel) $\quad \ldots \quad \ldots . \quad \ldots \quad \ldots \quad \ldots$

24 (Publication de la $3^{\text {eme }}$ Edition du Parallax $\begin{array}{lllllll}\text { Catalogue) } \quad \ldots & \ldots & \ldots & \ldots & \ldots\end{array}$

27 (Cartes d'Etoiles variables dans l'Hémisphère $\begin{array}{llllllllll}\text { sud) } \quad & \ldots & \ldots & \ldots & \ldots & \ldots & \ldots\end{array}$

27 (Traduction et Impression de l'̈̈ntroduction $\ddot{\mathrm{du}}$

Catalogue and Ephemerides) $\ldots$... $\ldots$. $\ldots$
ité Exécutif (Colloque organisé par l'U.A.I. et l'Union de Mécanique Théorique et Appliquée)... …

\begin{tabular}{cr} 
Dollars & Francs-0r \\
I63 & 500 \\
392 & 1,200 \\
327 & 1,000 \\
882 & 2,700 \\
980 & 3,000 \\
980 & 3,000 \\
261 & 800 \\
327 & 1,000 \\
4,409 & 13,500 \\
3,919 & 12,000 \\
3,266 & 10.000 \\
\hline 5,906 & 48,700
\end{tabular}

Dollars Francs-or

$\mathrm{I}, 960 \quad 6,000$

$816 \quad 2,500$

$2,6 \mathrm{r3} \quad 8,000$

$327 \quad x, 000$

$6,859 \quad 21,000$

I,633 5,000

$26 \mathrm{I} \quad 800$

26I $\quad 800$

$\frac{3,266}{\mathrm{r} 7,996} \quad \frac{\mathrm{ro,000}}{55, \mathrm{I00}}$

2. Que l'unité de cotisation pour les années de I949 jusqu'à la prochaine Assemblée Générale soit fixée à 500 francs-or.

* Les montants définitivement accordés comme subventions sont ceux exprimés en francs-or dans les résolutions de la Commission des Finances, le franc-or étant défini comme 0.2903225 grammes d'or fin, ou $\$ 0^{\circ} 3266$ au cours actuel. 